\title{
Regelung eines instationär betriebenen Festbettreakłors mit Fuzzy-Kontrollregeln
}

\author{
Dr.-Ing. U. Nieken, Ludwigshafen, Dipl.-Ing. G. Kolios und \\ Prof. Dr.-Ing. G. Eigenberger, Stuttgart
}

Katalytische Abluftreinigung in Reaktoren mit periodischer Strömungsumkehr erlaubt einen autothermen Betrieb bis zu niedrigen Schadstoflkonzentrationen. Bei höheren Schadstoffkonzentrationen ermöglicht ein Mittenabzug die vollständige Auskopplung der Verbrennungswärme [3].

Aufgabe der Regelung ist es, den gezündeten Zustand bei geringen Rohgaskonzenusationen durch Zugabe von Stützgas aufrechtzuerhalten und bei höheren Konzentrationen Reaktionswärme in Form eines heißen Teilgasstromes zurückzugewinnen.

Ein konventioneller Reglerentwurf gestaltet sich in diesem Fall als schwierig, da der Reaktor keinen definierten Arbeitspunkt besitzt, sondern nur im gezündeten Zustand gehalten werden muB, wobei er sich aufgrund der direkten Kopplung von chemischer Reaktion und Wärmetausch stark nichtlinear verhă]t.

Aus diesem Grund werden die Methoden der Fuzzy-Regelung ausgewählt und an einem Reaktor im Labormafstab erprobt. In folgenden wird zunächst kurz auf das Prinzip der katalytischen Oxidation mit periodisch wechselnder Strömungsrichtung eingegangen, um die Wahl der Reglerein- und Ausgangsgrößen zu begründen. Anschließend werden der Aufbau des Fuzzy. Reglers skizziert und die erzielten Versuchsergebnisse diskut iert.

\section{Aufgabenstellung}

Zur Reinigung von kalten Abluftströmen mit Spuren an organischen Verunreinigungen werden zunehmend katalytische Festbettreaktoren eingesetzt, bei denen die Strömungsrichtung periodisch umgekehrt wird [3]. Diese zunächst von Matros $[1,5,6]$ für die Schwefelsăureherstellung vorgeschlagene Fahrweise nutzt das Festbett sowohl als Regenerativ-Wärmetauscher als auch als Katalysatorträger. Gegenüber den konventionellen Verfahren, bei denen ein externer Wärmeübertrager und ein adiabater Fest bettreaktor gekoppelt werden, ist durch den integrierten regenerativen Wärmetausch bereits bei sehr kleinen Zulaufkonzentrationen ein autothermer Reaktorbetrieb möglich. Wie bei allen autothermen Verfahren ist es auch hier notwendig. mit Hilfe einer Regelung Sorge zu tragen, daß der gezündete Zustand in Zeiten geringer Schadstoffzufuhr erhalten bleibt. Darüberhinaus soll während Zeiten eines hohen Schadstoffangebots die Reaktionswärme möglichst vollständig zurückgewonnen werden.

\footnotetext{
'Dr Ing Ulrich Nieken, BASF-AG, Ludwigshafen, Dipl Ing Grigonos Kolios und Prof Dr Ing Gerhart Eigrnberger. Instifut fur Chemische Verfahrenstechnik der Universität Stultgart, Böhlınget Str. 72, 7000 Stuttgart 1
} 

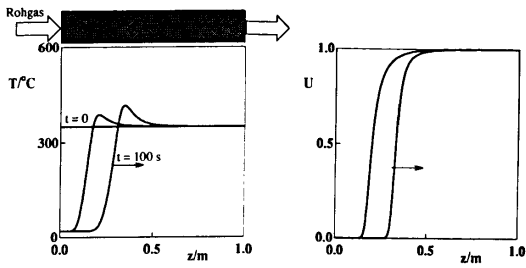

Abbildung 1. Temperatut - und I'msatzprofile zum Startzeitpunkt und $50 \mathrm{~s}$ bzw. 100 s nach dem Austıömen von lınks $T^{\prime \prime}=20^{\circ} \mathrm{C}, g^{\prime \prime}=0.1$ Gew.\% Propan.

\section{Prinzip des Verfahrens}

U'm in den gezündeten autothermen Betrieb zu gelangen muß der Reaktor zu Beginn auf ein Temperaturniveau vorgeheszt werden, bet dem das zu oxidierende Schadgas vollstăndig umgesetzt wird. Ist ein genügender Teil des Bettes auf Temperaturen oberhalb der Zündtemperatur des Schadgases aufgeheizt, wird der kalte Rohgasstrom in das Bett eingeleitet. Durch die im Festbett gespeicherte $W$ ärme wird das Gas aufgeheizt und nach Erreschen hinreichend hoher Temperaturen oxidiert. Allerdings führt die Zufuhr des kalten Roligases zu einer fortschreitenden Abkühlung des Bettanfangs und somit zu einer stetigen Verschiebung der Temperaturfront in Strömungsricht ung (Abb. I).

Um ein Verlöschen zn vermeiden, wird die Strömungsrichtung umgekehrt solange noch genügend Wärme im Festbett gespeichert ist. Durch das nun von rechts einströmende kalte Gas bildet sich eine weitere Temperaturfront aus, die sich, zusammen mit der zuerst erzeugten Temperaturfront, nach links durch den Reaktor bewegt (Abb. 2). Nach einer größeren Anzahl von Strömungsumkehrungen stellt sich im Reaktor ein zyklisch stationärer (_eingeschwungener “) Zustand ein (Abb. 3). $\mathrm{Er}$ ist dadurch gekennzeichnet, daB die während einer Periode freigesetzte Reaktionswärme gleich der vom Gas über den Reaktorrand abgeführten Wärme ist, so daß die Temperatur- und Umsatzprofile nach zwei aufeinanderfolgenden Umkehrungen der Strömungsrichtung identisch sind.

Abb. 3b zeigt den sägezahnförmigen Verlauf der Reaktoraustrittstemperaturen über der Zeit. Bei Anströmung von links nimmt die Temperatur am rechten Reaktorrand an Ende der Halbperiode ein Maximum an. Nach der Strömungsumkehr făllt die Temperatur rasch wieder bis auf die Zulauftemperatur ab. Das Integral des Wärmeaustrags über eine Pcriode entspricht im eingeschwungenen Zustand der während 

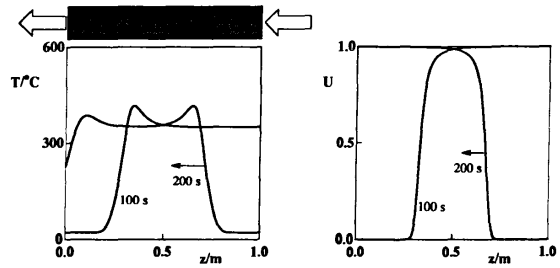

Abbildung 2: Temperatur- und Umsatzprofile zum Zeitpunkt der Strömungsumkehr nach 100 und $200 \mathrm{~s}$.

einer Periode freigesetzten Reaktionswärme [3].

Die Umschaltung der Strömungsrichtung kann entweder durch Ventile und Klappen geschehen, oder, nach dem Prinzip der Ljungst röm Wärmetauscher, durch Rotation eines segmentierten Festbettes bei feststehendem Ein- und Auslauf. Der Drehwinkel entspricht in diesem Fall der Zeit nach der Strōmungsumkehr.

Die nachfolgend dargestellten Experimente wurden an einer Anlage mit Ventilsteuerung durchgeführt. Das Anlagenschema ist in Abb. 4 dargestellt.

Kernstück der Anlage ist ein mit monolithischen Katalysatoren bestückter Reaktor, dem kalte Luft mit Spuren von Propan und/oder Propylen zugeführt werden. Für die Wărmerückgewinnung kann ein heißer Teilgasstrom aus der Reaktormitte entnommen werden. Zur Analytik stand ein CO-MeBgerät und ein Flammenionisationsdetektor zur Verfügung. Das Temperaturprofil wurde an 14 Stellen durch Thermoelemente vermessen. Die unvermeidlichen Wärmeverluste konnten nur zum Teil durch eine Begleitheizung kompensiert werden, um Rückwirkungen der Heizung auf das Reaktorverhalten auszuschließen.

\section{Auswahl von Reglerein- und -ausgangsgrößen}

Bei der katalytischen Abluftreinigung sind Störungen insbesondere durch Schwankungen von Konzentration und Zusammensetzung des Rohgases zu erwarten.

Anstelle der Rohgaskonzentration $g_{i}^{\circ}$ wird im folgenden die adiabate Temperaturerhöhung $\Delta T_{\text {od, }}$ jecier Schadstofikomponente des Gasstromes bei vollständiger Oxidation angegehen:

$$
\Delta T_{\text {ad. }}=\frac{\left(-\Delta h_{r, d}\right) / M_{,} \cdot g_{1}^{o}}{c_{p c}}
$$




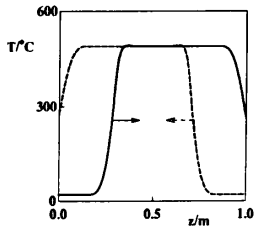

a)

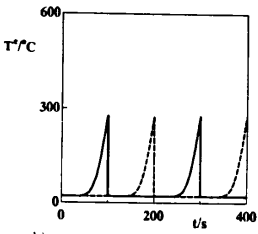

b)

Abbildung 3: Temperaturprofile im periodısch statıonăren Zustand unmittelbar vor Strömungsumkehr (a) und zeitlicher Verlauf der Aust rittstemperaturen (b).

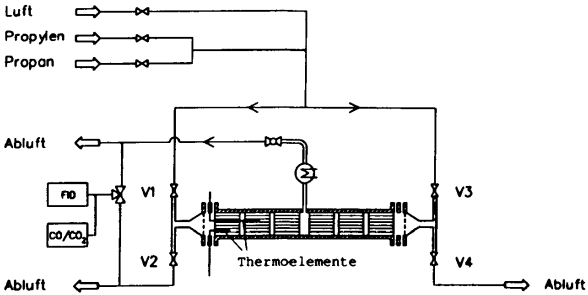

Abbildung 4: Das Schema der Versuchsanlage 


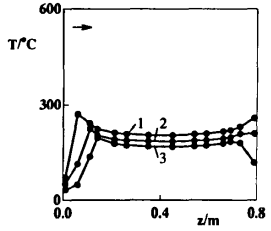

a)

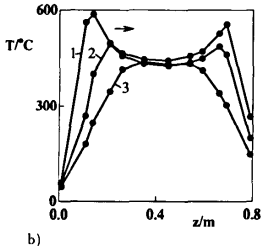

b)

Abbildung 5: Temperaturprofile im eingeschwungenen Zustand bei der Propylenoxidation (a) und der Propanoxidation (b).

1: $\Delta T_{a d}=120 \mathrm{~K}, 2: \Delta T_{a d}=70 \mathrm{~K}, 3: \Delta T_{a d}=30 \mathrm{~K}$, o Temperaturmeßstelle

Abb. 5 zeigt gemessene Temperaturprofile im eingeschwungenen Zustand unmittelbar vor Strömungsumkehr bei der Propylenoxidation (linkes Bild) und der Propanoxidation (rechtes Bild). Mit zunehmender Schadstoffkonzentration steigt, wie erwartet, die Maximaltemperatur an und die Temperaturfronten werden steiler. Bei gleichem Energieinhalt des Rohgases, d.h. gleicher adiabater Temperaturerhöhung. führt die Oxidation von Propan zu Reaktortemperaturen, die $200{ }^{\circ} \mathrm{C}$ über denen der Propylenoxidation liegen. Die Maximaltemperatur hängt also nicht nur vom Brennwert des Rohgases, sondern auch entscheidend von der Kinetik der Reaktion ab [3]. Sind beide Komponenten im Rohgas vorhanden, können drei stabile zyklisch stationäre Reaktorzustände existieren [7].

Bei einem Wechsel der Schadstoffusammensetzung kann es, trotz konstantem Heizwert des Rohgases zll eınem Absinken der Maximaltemperatur unter die Zündgrenze der Propanoxidation kommen. So bewirkt in Abb. 6 die sprungförmige Änderung der Zulaufzusammensetzung, ausgehend vom eingeschwungene Zustand mit hohen Propananteil zu einem Rohgas mit wenig Propan und hohem Propylenanteil. ein langsames Absinken der Reaktortemperaturen. Die zudosierte Propanmenge entsprechend einer adiabaten Temperaturerhöhung von $12 \mathrm{~K}$ reicht nicht zur Erhaltung des oberen gezūndeten Betriebspunktes aus, so daB das Temperaturprofil auf das Niveau der reinen Propylenoxidatıon abfällt und Propan unreagiert durchbricht.

Daraus folgt, daß der Gesamt-C-Gehalt im Zulauf, der sich online näherungsweise mit einem Flammenionisationsdetektor bestimmen ließe, als charakteristische Regelgröße nicht in Betracht kommt. Die Messung der Reingaskonzentration ist als Reglereingangsgröße ehenfalls ungeeignet. Zum einen wird eine vollständige $O x i$ dation angestrebt, so daB nur sehr kleine Regelabweichungen zugelassen werden können. Zum anderen kann der Fall eintreten. daß die Rohgaskonzentrationen der Schadstoffe aut null ahsinken. worauf der Reaktor ohne Stelleingriff verlöscht. Außerdem zeigt das Beispiel in Abb. 6, daB die Zulaufkonzentration der am schwersten 


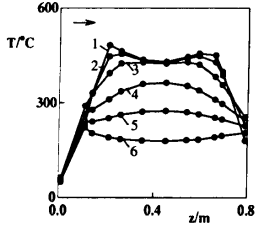

a)

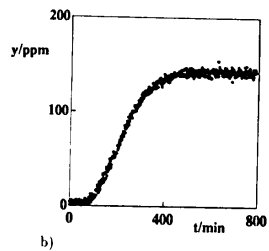

Abbildung 6: Übergangsverhalten des Reaktors nach einem Sprung in der Rohgaszusammensetzung von $70 \mathrm{~K}$ Propan. $0 \mathrm{~K}$ Propylen zu $12 \mathrm{~K}$ Propan und $58 \mathrm{~K}$ Propylen.

a) Temperaturprofil in eingeschwungenen Zustand bei $\Delta T_{\text {od }}$ (Propan) $=70 \mathrm{~K}(1)$ und (2) nach $7 \mathrm{~min}$. (3) nach $17 \mathrm{~min},(4)$ nach $170 \mathrm{~min}$, (5) nach 370 min nach dem Wechsel der Rohgaszusammensetzung. (6) neuer eingeschwungener Zustand.

b) Verlauf des FID-Signals über der Zeit (Kalibrierung mit Propan)

oxidierbaren Komponente nicht zu klein werden darf, weil sich sonst eine niedrigere Maxımaltemperat ur einstellt, be der dieser Schadstoff nicht mehr umgesetzt werden kann.

Daraus folgt, daß stets eine solche Maximaltemperatur im Reaktor aufrecht erhalten werden muB. daß der am chwersten oxidierbare Schadstoff noch sicher umgesetzt werden kann, sobald er un Zulauf vorhanden ist.

Dıe erste Regelgı̈ße ist somıt diese Maxınaltemperatur Sie läßt sich nur dadurch wirtschaft lich einstellen. daß ein Stützgas mit einer vergleichbaren oder etwas höheren Zündtemperatur eingesetzt wird. Diese Stützgaszufuhr stellt somit die entsprechende Stellgröße dar.

Am Temperatur- und Imsatzverlauf von Abb. 6 wird deutlich. daB die Stützgaszufuhr spätestens nach 100 min einsetzen müßte, um den Vollumsatz von Propan aufrechtzuerhalten.

\subsection{Mittenabzug}

Die im Ablaufstrom des Reaktors als fühlbare Wärme gespeicherte Reaktionsentha]pie kann wegen der im Mittel niedrigen und stark schwankenden Ablauftemperatur (Abb. 3b) meistens nicht weiter genutzt werden. Zur Energierückgewinnung auf hohem Temperaturniveau kann dagegen ein Teilgasstrom in der Reaktormitte abgezogen werden [2]. Abb. $i$ zeigt das eingeschwungene Temperaturprofil mit und ohne Mittenabzug. Durch den Mittenabzug kontrahiert das Temperaturprofil und 


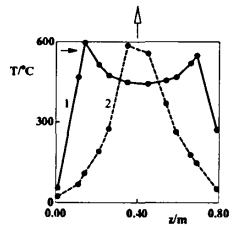

Abbildung $i$ : Einfluß des Mittenabzuges auf das Temperaturprofil. Eingeschwungener Zustand ohne (1) und mit $20 \%$ Gasalozug aus der Reaktormitte (2). $\Delta T_{\text {ad }}=$ $120 \mathrm{~K}$. Propanoxidation.

die Reaktoraust rittstemperatur sinkt auf das Niveau der Zulauftemperatur ab. Ist die Zone hoher Temperatur in der Reaktormitte breit genug, so wird im Mittenabzug gereinigte Luft abgezogen, und die Reaktionswărme vollständig als heißer Teilgasstrom zurückgewonnen. Allerdings kann Vollumsatz aller Komponenten bei Bet rieb mit Mittenabzug nicht mehr durch Einhaltung einer Maximaltemperatur allein gewährleistet werden, da die Strecke, auf der Maximaltemperatur herrscht, stark von dem Anteil des Mittenabzugs abhängt. Für die Regelung ist daher eine weitere Eingangsgrößc. not wendig.

\subsection{Geschätzter Umsatz der Reaktion}

I'm den vollständigen I'msatz aller Komponenten zu gewährleisten, muB das Gas hinreichend lange in einem Bereich hoher Temperaturen verweilen. Wie hoch die Temperaturen sein müssen, wird mabgeblich durch die Reaktionsgeschwindigkeit der am schwersten zu oxidierenden Komponente bestimmt. Im folgenden wird der Uimsatz dieser am schwersten oxidierbaren Komponente aus dem gemessenen Temperaturprofil über der Schüttungslänge abgeschätzt und der geschätzte Umsatz als weitere Reglereingangsgröße benutzt.

Dazu wird für diese Komponente von einer Reaktion 1. Ordnung ausgegangen. StoBfaktor $k^{\infty}$ und Aktivierungsenergie $E$ miissen in getrennten Versuchen vorab bestimmt werden. Die Massenbilanzen für diese Komponente in Feststoff und Gas sind im Vergleich zur Energiebilanz quasistationär und lauten (eindimensionales Zweiphasenmodell) :

$$
\begin{aligned}
& B \cdot a_{v} \cdot\left(c-c^{*}\right)=a_{v} \cdot k^{\infty} \cdot e^{-\frac{E}{\hbar t}} \cdot c^{*} \\
& \frac{\dot{m}}{A} \cdot \frac{d g_{t}}{d z}=-M_{1} \cdot \beta_{1} \cdot a_{v} \cdot\left(c_{2}-c_{1}^{*}\right)
\end{aligned}
$$


Mit den Imrechnungen

$$
c_{\mathrm{i}}=g_{\mathrm{t}} \cdot \frac{\varrho_{G}}{M_{\mathrm{t}}} \quad, \quad \varrho_{G}=\frac{p \cdot M}{R \cdot T} \approx \frac{p \cdot M_{L}}{R \cdot T}, \quad k(T)=k^{2} \cdot \mathrm{e}^{-\frac{E}{R T}}
$$

läßt sich Gl. 2 nach $c^{*}$ auflösen und in (il. 3 cinsetzen. Durch Integration über die Reaktorlänge läßt sich der Endumsatz $l^{*}=1-g^{*} / g^{*}$ wle folgt au dem Temperaturprofil im Reaktor herechnen.

$$
U^{\prime}=1-\exp \left(-a_{2} \cdot \frac{A}{\dot{m}} \cdot \int_{z=0}^{z=L} \frac{M I_{L} \cdot p}{R \cdot T(z)} \cdot \frac{h(T(z)) \cdot 3}{k(T(z))+3} d z\right)
$$

Interpoliert man aus den gemessenen Reaktortemperaturen ein Temperaturprofil $T(z)$. so läßt sich bei bekanntem StoBfaktor $k^{2}$ und Aktiverungenerge $E$ eine Schätzung für den l'msatz der am schwersten zu oxidierenden Komponente durchführen.

Im Laborreaktor wurden die Parametes $k^{x}$ und $E$ identifiziert, inden von enem stabilen gezündeten Propanbetrıeb die Zulaıfkonzentration von Propan soweit abgesenkt wurde, daß die Wärmefreısetzung nicht mehr zum Erhalt des gezündeten Zustandes ausreichte. Ahb. 8 zeigt die Temperaturprofile zu verschiedenen 7eit punkten und den zeitlichen Verlauf von Maximaltemperat ur und Aust ritiskonzentration Wăhrend die Maxımaltemperat ur zunächst sehr schnell absinkt, kommt es erst nach ca. 5 Stunden zum Durchbruch des Schadstoffes. Mit Hilfe der gemessenen Tempe. raturprofile und Gl 4 wurden die Paranater der Reaktionsgeschwindigkeit an den gemessenen I'msatzverlauf angepaßt.
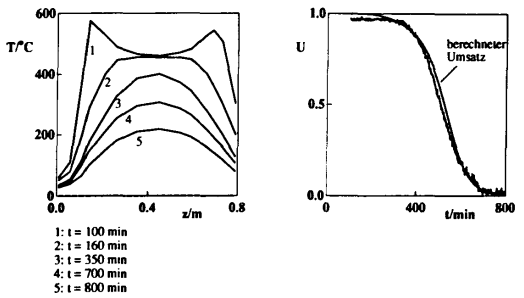

Abbildung 8: Zur Parameteranpassung für die Umsatzschātzung. links: die gemessenen Temperaturprofile rechts: gemessener und durch Anpasung von $k^{\infty}$ und $E$ berechneter Imsatz 
An dieser Stelle sei angemerkt, daB auch eine grobe Approximation der Reaktionskinetik für die nachfolgend dargestellte Regelung ausreicht, da mit Hilfe der so ermittelten Reaktionskinetik lediglich der Temperaturverlauf im Reaktor bewertet (gewichtet) wird.

\section{Der Fuzzy-Regler}

Mit den beiden Eingangsgrōßen Maximaltemperatur und geschātztem Umsatz lāßt sich der Zustand des Reaktors charakterisieren. Ziel ist es, möglichst sparsam St ützgas zu dosieren und, wenn möglich, Reaktionswärme in Form eines heißen Seitenstromes zurïckzugewinnen. Im Gegensatz zu vielen konventionellen Regelaufgaben ist es aber nicht Aufgabe der Regelung, einen genau definierten Zustand im Reaktor einzustellen, vielmehr soll lediglich der gezündete Zustand erhalten bleiben und ggf. ein Optimum an Reaktionswärme ausgekoppelt werden.

Wegen dieser nur qualitativ zu begründenden Anforderungen der Regelungsaufgabe lag es nahe, mit der Fuzzy-Regelung ein auf qualitativer Bewertung beruhendes Regelungskonzept einztisetzen.

Übliches Vorgehen beim Entwurf eines Fuzzy-Reglers ist die Definition von Regelund Stellgrößen als "linguistische Variable" und die Beschreibung der Regelaufgabe durch verbale Kontrollregeln [8].

Als Eingangsgrößen dienen die Maximaltemperatur und der geschätzte Umsatz bzw. dessen zeitliche Änderung, als Stellgrößen der Stützgasstrom und der abgezogene Seitenstrom bzw. deren Änderungen.

Für den Regler wurden somit folgende linguistische Variablen gewählt:

$\begin{array}{ll}\text { Maximaltemperatur } & \left(T_{\max }\right) \\ \text { Abweichung vom Sollumsatz } & (\Delta U) \\ \text { Änderung der Stützgasmenge } & (\Delta S T) \\ \text { Änderung des Seitenstromes } & (\Delta M I)\end{array}$

Die Ein- und Ausgangsgrößen werden für die Fuzzy-Regelung zunächst auf einen Bereich von $(-10$ bis +10$)$ normiert, wobei dieser Bereich fünf verschiedenen $A$ usprägungen (PB positiv big. PS positiv small, $Z$ zero, NS negative small, NB negative big) der linguistischen Variablen zugeordnet wird. Die Festlegung auf fünf Ausprägungen pro lınguistischer Variable ist dabei willkürlich und wurde aus [9] übernommen.

Die Regelungsaufgabe wird nun in sogenannten Kontroliregeln hinterlegt. In diesem Fall wurden zwei Regelsătze (für Stützgas und Mittenabzug) mit je 25 Regeln (2 Eingangsgrößen $\times 5$ Ausprägungen) aufgestellt.

Beispielhaft sei die folgende Regel herausgegriffen:

Wenn die Marimaltemperatur sehr gro $\beta$ ist $(P B)$ und der Umsatz geringfügıg zuntmmt (PS) dann eoll der .1tuttenabzig stetig um einen kletnen Faktor vergrößert ueruten (PS). 


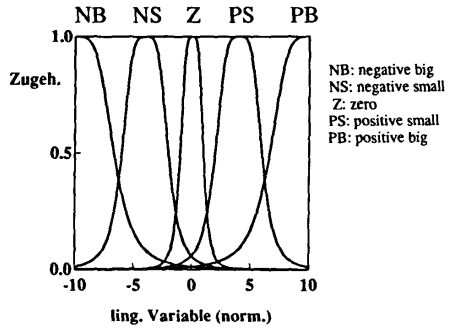

Abbildung 9: Zugehörigkeitsfunktionen der Eingangsgrößen

In formaler Schreibweise:

Wenn $\left(\left(T_{\max }=\mathrm{PB}\right)\right.$ und $\left.(\Delta U=\mathrm{PS})\right)$ dann $(\Delta M=\mathrm{PS})$

Diese Regel beschreibt einen integrierenden Regler für die Stellgröße Mittenabzug.

Um die Regeln auswerten zu können, müssen zunächst die Zugehörigkeiten der lınguistischen Variablen zu den Ausprägungen festgelegt werden. Eine hier benutzte Zuordnung ist in Abb. 9 dargestellt. Sie folgt aus einem Vorschlag von [4], nach dem die Zagehörigkeiten der Eingangsgrößen durch folgende Funktionen beschrieben werden

$$
\mu(x)=\frac{1}{1+(a(x-c))^{b}}
$$

Der Parameter $c$ verschiebt das Maximum der Funktion, ein großer Wert von $a$ zieht die Wendepunkte näher an das Maximum, $b$ ändert die Steilheit der Flanken.

Abb. 9 zeigt die Zugehörigkeitsfunktionen für die Eingänge. Ein normierter Wert der Maximaltemperatur von +9 ergibt, wie aus Abb. 9 abzulesen, eine Zugehörigkeit zu PB von 0.99 und zu PS von 0.025.

Die Zuordnung der Ausprägungen zu den entsprechenden Werten der Ausgangsgrößen (Änderung Stūtzgasmenge, Ānderung Seitenstrom) erfolgte ūber Dreiecksfunktionen.

Für die Verknüpfung von linguistischen Variablen wurde der Minimum-Operator für die ."und"-Verknüpfung und der Maximum-Operator für die noder *-Verknüpfung gewählt. Ist also die Aussage ${ }_{n}\left(T_{\text {mox }}=\mathrm{PB}\right)^{\star}$ zu 0.9 erfült und ${ }_{n}(\Delta U=\mathrm{PS})^{\mu}$ zu 0.5 , dann ist die Alussage $-\left(T_{\text {mex }}=P B\right)$ und $\left(\Delta I^{t}=P S\right)^{*}$ zu 0.5 . die Aussage ${ }_{n}\left(T_{\max }=\right.$ $\mathrm{PB}) \operatorname{oder}\left(\Delta I^{\circ}=\mathrm{PS}\right)^{-}$zu 0.9 erfïllt. 
In der Fuzzy-Logic geht man nun davon aus, daB dic SchluBlolgerung einer Regel zum gleichen Grad erfüllt ist wo die Vorbedingung. d.h. im o.a. Beisplel ist die SchluBfolgerung ${ }_{n} \Delta M=\mathrm{PS}^{-}$zu 0.5 erfüllt. Durch Auswert ung weiterer linguistıscher Variabler wird jeder Ausprägung der linguistischen Ausgangsvariablen ein Erfülltheitsgrad zugeordnet. Damit ergibt sich beispielsweise $\mu(\Delta . M=P S)=0.5$, $\mu(\Delta . M=\mathrm{PB})=0.1, \mu(\Delta . M=Z)=0.1$ etc. Nun muß dieser unscharfen Menge wieder ein realer Zahlenwert der Ausgangsgröße zugeordnet werden. Dazu wırd jede Auspıăgung der Ausgangsgröße mit ıhrem Erfülltheitsgrad multipliziert, so daß eine gewıchtete Fläche resultiert (Abb. 10). Dieser Schritt wird als Inferenz bezeichnet. Aus dıeser gewichteten Fläche muB nun noch ein konkreter Wert ausgewählt werden, beispıelsweise die Abszisse des Flächenschwerpunktes. Man spricht dabei von Defuz?:fizierung.

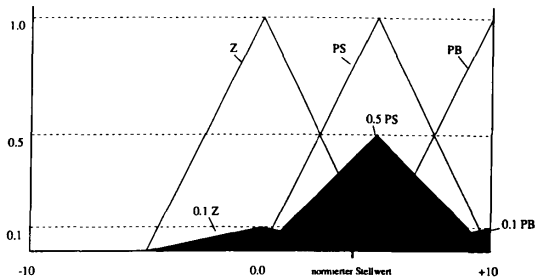

Abljildung 10: Zur Bestımmung der Ausgangsgröße (,normierter Stellwert*) durch Inferenz und Defuzzyfizierung

Ziel ist auch hier mur, daß das als intuitiv ,vernünftig ${ }^{\star}$ empfundene Vorgehen zutreffend wiedergegeben wird.

\section{Versuchsergebnisse}

Die nachfolgend dargestellten Versuche basieren auf einer Regelbasis, die aufgrund allgemeiner Betriebserfahrung aufgestelit und nicht weiter optimiert wurde.

Als Störgröße wurde die Propankonzentration im Zulauf entsprechend Abb. 11 variiert. Auf eine Zudosierung von Propylen wurde verzichtet, da Propylen das Temperaturprofil kaum verändert, solange Propan sicher oxidiert werden kann (siehe Abb. 6). Ein Schadst offstrom von $10 \mathrm{~g} / \mathrm{h}$ Propan ( $=80 \mathrm{~K} \Delta T_{\text {add }}$ ) reicht für einen autothermen Reaktorletrieb aus, erlaubt aber keine zusätzliche Wärmeabfuhr. Bei $20 \mathrm{~g} / \mathrm{h}\left(\Delta T_{\text {ad }}=160 \mathrm{k}\right)$ kann ein heißer Seitenst rom abgezogen werden. 


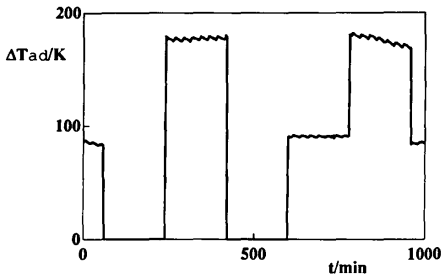

Abbildung 11: Verlauf der Störgrößle (Propankonzentration)

Abb. 12 zeigt den Verlauf der Maximaltemperatur, die Ausgangskonzentration und den zeitlichen Verlauf der Stellgrößen Stützgaseinspeisung und Mittenabzug. Die sprungförmige Absenkung der Rohgaskonzentration auf Null nach $60 \mathrm{~min}$ wird durch Zugabe von Stützgas (Propan) ausgeregelt. Die Maximaltemperatur bleibt bei etwa $500^{\circ} \mathrm{C}$, und die Austrittskonzentration liegt unter $5 \mathrm{ppm}$ Propanäquivalente. Etwas unbefriedigend ist die Oszillation der zugegebenen Stützgasmenge, die sich auch in kleinen Oszillationen der Maximaltemperatur wiederfindet.

Nach einem starken Anstieg der Schadstofikonzentration schließt das Stützgasventil und mit einer leichten Verzögerung offnet sich der Mittenabzug. Damit geht einerseits ein Zusammenzichen des Temperaturprofils einher (siehe Abb. 7 ), andererseits steigt die Maximaltemperatur aufgrund der großen Zulaufkonzentration an.

Eın erneutes Absinken der Rohgaskonzentration bei einem schmalen und hohen Temperaturprofil im Reaktor (der Mittenabzug ist geöffnet) führt zu einer geringeren Stiutzgaszugabe solange die Maximaltemperatur und damit auch der geschätzte I'msatz hoch sind.

Nach einem Anstieg der Propanmenge auf $10 \mathrm{~g} / \mathrm{h}\left(\Delta T_{\text {ad }}=80 \mathrm{~K}\right)$ schlieBt die Stützgaszufuhr. ein weiterer Anstieg der Rohgaskonzentration führt zur erneuten Öffnung des Mittenabzugs.

Von zwei Messungen abgesehen bleibt die Austrittskonzentration wăhrend des Versuchs immer deutlich unter $10 \mathrm{ppm}$. das entspricht einem Umsatz von $99.9 \%$.

Zusammenfassend läBt sich sagen, daB der Regler ohne jegliches Tuning zufriedenstellend arbeitet, inshesondere was die Reingaswerte betrifft. Lediglich die Stützgaszudosierung berlarf der Optimierung. um Schwankungen der Stellgrößen zu vermindern. 

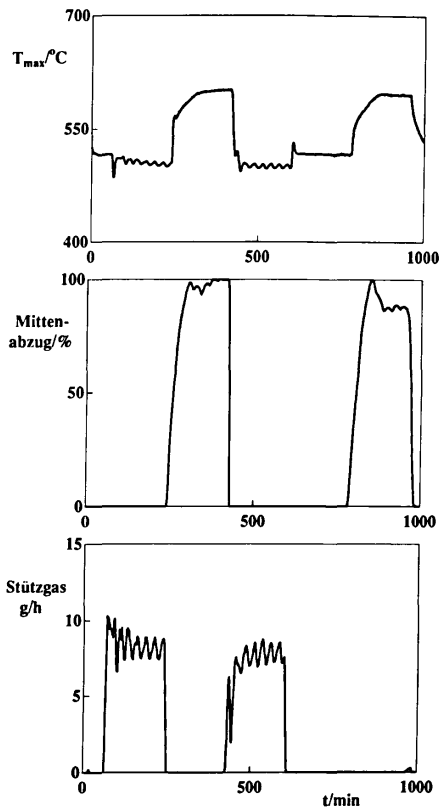

Abbildung 12: Verlauf der Maximaltemperatur (oben), des Mittenabzuges (Mitte) und der Stïtzgaseinspeisung (unten) bei Störung gemäß Abb. 11. 


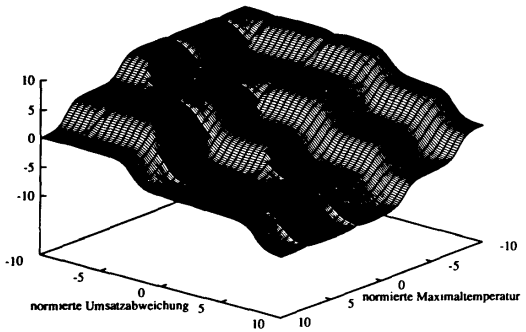

Abbildung 13: Die Kennfläche der Stellgröße Stützgaseinspeisung über den normierten Eingangsgrößen

\subsection{Monotonie}

In dem hier vorgestellten Beispiel verstellen zwei Eingangsgrößen, der geschätzte Umsatz und die Maximaltemperatur in Form einer Kennfeldregelung, zum einen die Stützgaszufuhr zum andern den Mittenabzug. Bei einer derart einfachen Konstellation kann die durch die linguistischen Regeln definierte Kennfläche direkt dargestellt werden. Abb. 13 zeigt die Änderung des Stützgasstromes als Funktion der Eingangsgrößen. Wie erwartet ergibt sich ein nichtlinearer Zusammenhang mit - bedingt durch die Form der Zugehörigkeitsfunktionen - runden Übergängen. Zunächst überraschend ist aber, daß die so definierte Kennfläche nicht monoton ist, sondern Einschnitte aufweist. Der Grund dafür soll an einem einfachen Beispiel aufgezeigt werden.

Angenommen man hat fĩr ein System mit einem Eingang $E$ und einem Ausgang $A$ die folgenden drei Regeln definiert:

$$
\begin{array}{llll}
(E=N B) & \rightarrow & (A=Z) & \text { Regel 1 } \\
(E=Z) & \rightarrow & (A=P B) & \text { Regel 2 } \\
(E=P B) & \rightarrow & (A=P B) & \text { Regel 3 }
\end{array}
$$

mit den in Abb. 14 dargestellten Zugehörigkeitsfunktionen.

Für den Zusammenhang zwischen Ein- und Ausgangsgrōßen könnte man erwarten, daß der Ausgang gleich null ist ( $Z$ zero), falls der Eingang klein ist (NB negativ big) und bei größeren Werten die Ausgangsgröße zunimmt (Regel 2, Regel 3). 


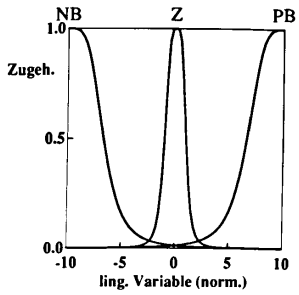

Abbildung 14. Zugehörıkkeitsfunktıonen

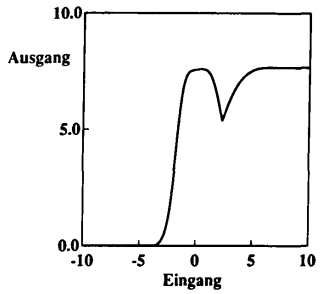

Abbiklung 15: Kennlinie der Ausgangsgröße 


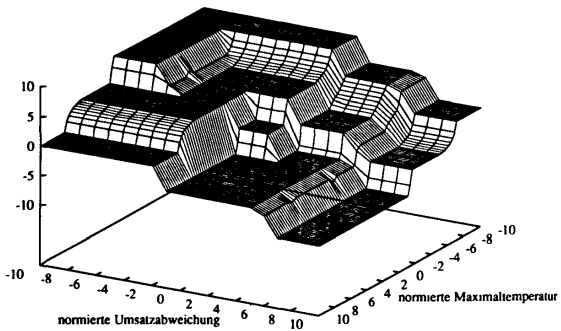

Abbildung 16: Kennfläche der Stellgröße Stützgaszufuhr über den normierten Eingangsgrößen bei trapezförmigen Zugehörigkeitsfunktionen

Tatsächlich ergibt sich der in Abb. 15 dargestellte Zusammenhàng mit einem relativen Minimum bei 2, also zwischen $Z$ (zero) und PB (positiv big). Grund für dieses Minimum ist, daß im Bereich der Eingangsgröße zwischen 2 und 4 weder Regel 2 noch Regel 3 in hohem Maße erfüllt sind und Regel 1 daher das Gesamtergebnis beeinfluBt.

Um derartige Überlagerungen sicher zu vermeiden, kann man entweder nur lokal defnierte Zugehörigkeitsfunktionen benutzen ( $\mathrm{Abb}$. 16) oder versuchen beim Aufstellen der Regeln sorgfältiger vorzugehen. Abb. 17 zeigt das Kennfeld für die Stützgaszudosierung basierend auf einem modifizierten Regelsatz mit nur 14 Regeln.

Generell ist darauf zu achten, dab immer eine oder wenige Regeln dominieren sollten. Ferner sollten Nichtlinearitäten besser in den Regeln als in den Zugehörigkeitsfunktionen hinterlegt werden.

\section{Zusammenfassung}

Der sichere Betrieb eines autothermen Festbettreaktors für die katalytische Abluftreinigung erfordert ein Regelungskonzept, das ein Verlöschen des Reaktors oder die Einstellung eines mittleren Betriebszustandes, bei der eine schwer oxidierbare Komponente nicht mehr vollständig urngesetzt werden kann, sicher vermeidet.

Im vorliegenden Brispirl sollte zusätzlich, wann immer möglich, überschüssige Reaktionswärme als heißer Mittenabzug ausgekoppelt werden. 


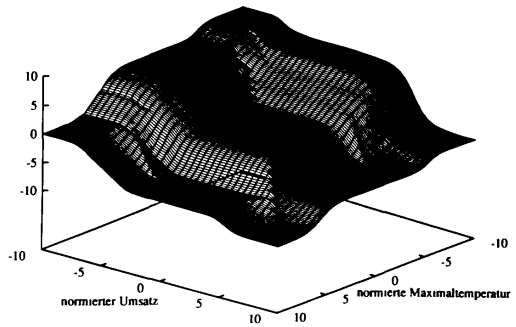

Abbıldung 17: Kennfläche der Stellgröße Stützgaszufuhr über den normierte , Eingangsgrōßen bei modifiziertern Regelsatz

Es zeigte sich. daß diese Aufgabe erfüllt werden kann, wenn als Regelgrößen die Maximaltemperatur sowie der aus dem akt uellen Temperaturprofil berechenbare fiktive Limsatz der am schwersten oxidierbaren Komponente gewählt werden. Stellgrößen sınd die Stützgaszufuhr, solange dieser U'msatz nicht ohne zusätzliche Energiezufuhr sichergestellt werden kann, sowie der Mittenabzug, falls ein C'berschuB an Reaktionswärme vorliegt.

Da beide Stellgrößen direkt auf eine Strecke mit großen Zeitkonstanten wırken, kann eine integruerende Regelung vorgesehen werden. Ihre Auslegung gestaltet sich jedoch als schwierig wegen der stark nichtlinearen Verkopplung, der möglichen C̈berlappung beider Stelleingriffe und der Tatsache, daß kein fester Arbeitspunkt eingestellt sondern nur ein qualitativ formuliertes Ziel erreicht werden soll.

Daher wurde eine Kennfeld-Regelung mit Hilfe von Fuzzy-Kontrollregeln entworfen und an einer Versuchsanlage erprobt. Dabei zeigte sich, dab sich schon mit einfachen Strukturen und Kontrollregeln befriedigende Ergebnisse erzielen lassen. Die weitgehend heuristische Vorgehenswerse erlaubt ein einfaches U'msetzen von Erfahrungswissen in Kontrollregeln. Allerdings ist es erforderlich und bei nicht zu komplexen Systemen auch gut durchführbar, die erhaltenen Kennfächen der Stellgrößen auf Konsistenz und Stetigkeit zu überprüfen. 


\section{Formelzeichen}

\begin{tabular}{|c|c|c|}
\hline$A$ & $\mathrm{~m}^{2}$ & Reaktorquerschnitt \\
\hline$a_{\mathrm{t}}$ & $\mathrm{m}^{2} / \mathrm{m}_{\text {Reaktor }}^{3}$ & spezifische geometrische Oberfläche \\
\hline$r$ & $\mathrm{kmol} / \mathrm{m}^{3}$ & Konzentration \\
\hline$c_{p G} G$ & $\mathrm{~kJ} /(\mathrm{kg} \mathrm{K})$ & spezifische Wärmekapazität des Gases \\
\hline$E$ & $\mathrm{~kJ} / \mathrm{kmol}$ & Aktivierungsenergie \\
\hline$g$ & & Massenanteil \\
\hline$C: z$ & $\mathrm{~kg} /\left(\mathrm{m}^{2} \mathrm{~s}\right)$ & Querschnittsbelastung \\
\hline$\vec{b} h_{r}$ & $\mathrm{~kJ} / \mathrm{kmol}$ & Reaktionsenthalpie \\
\hline$k^{x}$ & $\mathrm{~m} / \mathrm{s}$ & Stoßfaktor \\
\hline$k$ & $\mathrm{~m} / \mathrm{s}$ & Reaktionsgeschwindigkeitskonst ante \\
\hline$L$ & m & Katalysatorbettlãnge \\
\hline$\dot{m}$ & $\mathrm{~kg} / \mathrm{s}$ & Massenstrom \\
\hline$M$ & $\mathrm{~kg} / \mathrm{kmol}$ & molare Masse Schadstoff \\
\hline$M_{L}$ & $\mathrm{~kg} / \mathrm{kmol}$ & molare Masse Luft \\
\hline$p$ & $\mathrm{~Pa}$ & Druck \\
\hline $\boldsymbol{R}$ & $\mathrm{kJ} /(\mathrm{kmol} \mathrm{K})$ & allgemeine Gaskonstante \\
\hline$r$ & $\mathrm{kmol} /\left(\mathrm{m}^{2} \mathrm{~s}\right)$ & $\begin{array}{l}\text { Reaktionsgeschwindigkeit, bezogen auf } \\
\text { die äußere geometrische Oberfläche }\end{array}$ \\
\hline$T$ & K. ${ }^{\circ} \mathrm{C}$ & Temperatur \\
\hline$\frac{\Delta T_{\text {ad }}}{U}$ & $\mathrm{~K},{ }^{\circ} \mathrm{C}$ & $\begin{array}{l}\text { adiabate Temperaturerhöhung } \\
\text { Umsatz }\end{array}$ \\
\hline$z$ & m & axiale Ortskoordinate \\
\hline$B$ & $\mathrm{~m} / \mathrm{s}$ & Stoffübergangskoeffizient \\
\hline$\mu$ & & Zugehörigkeit \\
\hline$\rho_{G}$ & $\mathrm{~kg} / \mathrm{m}^{3}$ & Dichte Gas \\
\hline$e$ & Index & Reaktorende \\
\hline 2 & Index & Stoff \\
\hline$o$ & Index & Reaktoreintritt \\
\hline * & Index & Katalysatoroberfläche \\
\hline
\end{tabular}

\section{Literatur}

[1] G.K. Boreskov und Y.S. Matros. Unsteady-state performance of heterogeneous catalytic reactions . Catal. Rev.-Sci. Eng., 25(4):551-590, 1983.

[2] G. Eigenberger und U. Nieken. Catalytuc combustion with periodic flow reversal. Chemical Engineering Science, 42:2109-2115, 1988.

[3] G. Eigenberger und U. Nieken. Katalytsche Abluftresnigung: Verfahrenstechnische Aufgaben und newe Lösungen. Chemie Ingenieur Technik, 63(8):781-791, 1991 . 
[A] W. J. M. Kickert und H. R. Van Nauta Lemke. Applicatıonof a Fuzzy Controller in a Warm Water Plant. Automatica, 12:301 ff., 1976.

[5] Y. S. Matros. L'nsteady-state ondation of sulphur dioride for sulphuric actd production. Sulphur, (183):23-29, 1986.

[6] Y. S. Matros. Catalytic processes under unsteady-state conditions, Band 43 von Studies in Surface Science and Catalysis. Elsevier, 1989.

[7] $[$ : Nieken. Abluftreinigung in katalytischen Festbettraktoren bet periodischer Strömungsumkehr. Dissertatıon. I'nuversität Stuttgart, 1993.

[8] Thomas Tilli. Automatisierung mit Fuzzy-Logic. Franzis-Verlag, 1992.

[9] Y. Yamashita, S. Mlatsumato und M. Suzuki. Start-l'p of a ('atalytic Reactor by Fuzzy Controller. Journal of Chemical Engineering of Japan, 21(3):277 ff., 1988. 